\title{
Sum of Lambda Z Timepoints
}

National Cancer Institute

\section{Source}

National Cancer Institute. Sum of Lambda Z Timepoints. NCI Thesaurus. Code C85816.

The number of time points used in computing Lambda $\mathrm{z}$. 